\title{
Memory Virtualization Technique for Efficient Access of Data Resources in Cloud Environment
}

\author{
Pankaj Lathar \\ Research Scholar, Dept. of \\ Computer Science \& \\ Engineering \\ University Institute of \\ Engineering \& Technology, \\ M.D. University, Rohtak- \\ 124001
}

\author{
Yudhvir Singh, Ph.D \\ Associate Professor, Dept. of \\ Computer Science \& \\ Engineering \\ University Institute of \\ Engineering \& Technology, \\ M.D. University, Rohtak- \\ 124001
}

\author{
Girish Kumar Sharma, \\ Ph.D \\ Associate Professor\& Head, \\ P.G. Dept. of Computer \\ Science \\ Bhai Parmanand Institute of \\ Business Studies, Shakarpur, \\ Delhi 110092. (Govt. of NCT \\ Delhi) GGSIP University
}

\begin{abstract}
Working in cloud environment and accessing its services is fascinating task. It is one of modern technological areas to work upon it for development of nation and increase economy rate. The paper deals with improving desktop cloud computing services with the help of technique called Memory Virtualization. Since virtualization is efficient to extent scalability of typical desktop-as-a-service and make it suitable for Wide Area Network (WAN's) environments.

Desktop-as-a-Service (DaaS) in cloud computing means that user can access its own desktop services as well as services of other desktops located on remote servers. Memory virtualization not only works with virtual memory but it also maps physical memory instructions to actual machine memory. The instructions stored in machine memory are checked with incoming user client requests. Similar matched queries will result in generation of data using one of recovery techniques called Shadow Paging Technique. Data is produced and stored in remote cloud environment where user can access the data to perform their tasks.

The proposed systematic model for accessing desktop services is shown in following paper by using memory virtualization technique. A comparison is also made between paravirtualization technique and memory virtualization technique that gives solution in favor of memory virtualization technique.
\end{abstract}

\section{Keywords}

Cloud computing, Desktop-as-a-Service (DaaS), virtualization, memory virtualization, shadow paging

\section{Literature Survey}

Continuous efforts in field of cloud computing by various researchers and scholars have made this area as one of legend in IT sector. It is known that for using multiple virtual machines to access data also requires management of data and its collection. Its resources must be managed in optimal way in accordance with cloud providers service laws and agreements. Few data accessing and resource management techniques are shown below:

- Workload prediction [1]: - It is technique of predicting expected workload on respective servers in form of number of requests. It requires performance model to calculate impact of various problems associated with system.

- Memory reusing mechanism [2]:- It is used to decrease amount of data transferred through live virtual machine system. It is dynamic process that why we can reuse the data resources, thus leading to shorter migration time and processing time.

- Linear Scheduling Tasks and Resources (LSTR) [3]: - It is based on scheduling algorithms used in selecting criteria of operating system. The resources are distributed on basis of their priority and precedence level in cloud environment. It is done by selecting Quality of Service (QoS) parameters like cost, on demand etc. The resources having more demand will be processed first.

\section{INTRODUCTION}

The concept of cloud computing was originated from telephony networking scheme. Before evolution of cloud computing, there used to be virtual networks that perform work of connecting multiple computers. Since virtual networks makes task easier and compact, so this concept is broaden into separate technique called as Virtualization. With the help of virtualization, one server can act as multiple servers/operating systems that distributes task into cloud segments viz Application, Data and Connectivity. In this way, task is divided into number of sub tasks and all these tasks are combined to provide optimal solution.

Our aim is to access data resources from cloud at minimal rate and without violating service level agreements. Introducing virtualization technique in accessing will definitely prove beneficial but it is our task to use which type of virtualization. There are several types of virtualization like Full virtualization, Hardware virtualization, Application virtualization and many more [4]. Typical desktop-as-aservice is based on physical servers that employ tasks after processing requests one by one. It leads to wastage of time and large response time. So, both these factors are harmful for increasing system performance. The traditional desktop interface uses hardware and software installed tools. Without installing this software, we cannot access the required services. But concept of virtual desktop clouds removes this ambiguity of installation at various servers. Cloud computing is combination of various technologies like Grid computing, Virtualization, Autonomic computing, Ubiquitous computing, P2P computing and many more

The paper is divided into followings sections: Section 2 deals with concept of virtualization and its importance in cloud building environment. It also gives brief overview of memory virtualization technique. Section 3 presents proposed 
systematic architecture model for data accessing using memory virtualization. The data is transferred from machine memory to virtualization cloud environment with the help of shadow paging technique. It also tells how this technique is used to recover data from cloud in case of failure of system.

\section{ROLE OF VIRTUALIZATION IN CLOUD SECTOR}

To overcome tough and time consuming tasks in less time is one of factor that takes virtualization to great height. In 1990's, there used to be single server systems that takes huge time in computation of resources. They may even take years to process one request. With advent of time, things and technology keeps on growing. More complex environments have come that requires use of distributed information resources among several levels of abstraction. The only way to distribute resources evenly is availability of centralized data servers in cloud environments. So these conditions introduce the need of virtualization.

Virtualization is defined as technique to access data on multiple operating systems or servers in lieu of single physical server. We can say that single operating system is connected to multiple Virtual machines for performing tasks. Virtualization hides physical characteristics of resources from their clients. It hides characteristics because it is treated as like black box. Users are concerned about using these services instead of knowing how they originate into cloud. That's why cloud is sometimes called as Black Box.

This technique requires use of virtual managers called as Hypervisors for controlling flow of data coming through various incoming requests. Hypervisors are proven best for management of resources so that all clients can access data. They are set of programs that manage multiple operating system or multiple instances of single operating system. They manage CPU speed, memory and allocate resources according to operating system specifications. The three key elements viz cheap cost, easy accessible and reliability have made concept of virtualization to get included in every IT industry.

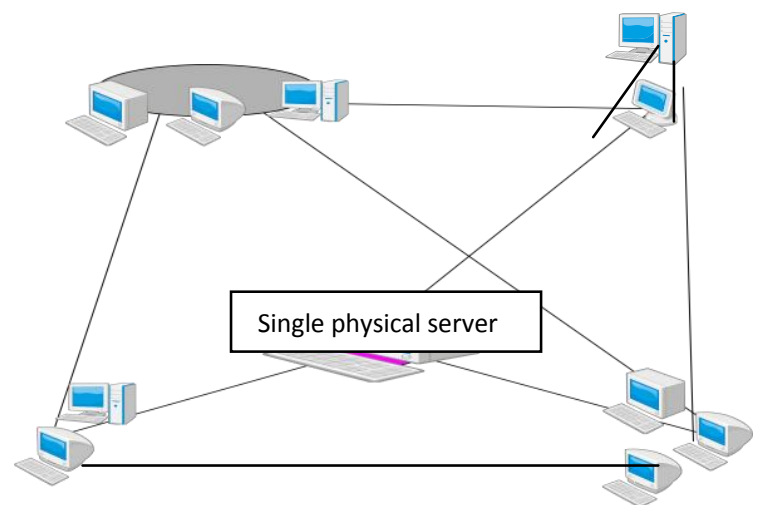

Fig 1: Virtualization Process where single physical server is situated in middle and it is connected to virtual machines for performing tasks faster.

\subsection{Memory Virtualization Technique}

The technique runs multiple virtual machines on single system where virtual management manager is responsible for mapping physical memory to actual machine memory. For providing mapped instructions to cloud, it uses Shadow paging technique which is elaborated in section 3 of this paper. It also provides provision to recover data from cloud in case of system failure.

Para-Virtualization technique: - This technique is also one of best methods to access data from cloud by modifying operating system kernel to virtualized instructions. Paravirtualization involves modifying the OS kernel to replace non-virtualizable instructions with hyper calls that communicate directly with the virtualization layer hypervisor. Para-virtualization is different from full virtualization where the unmodified OS does not know it is virtualized and sensitive OS calls are trapped using binary translation.

Are OS-Level Virtualization and Operating System Virtualization techniques same?

These two techniques often create confusion among users. They are defined as follows:

- OS-Level Virtualization:- This technique works on OS layer that is physical layer. The physical server instances are partitioned into multiple isolated portions where each partition represents single server. The OS kernel will run a single operating system and provide that operating system functionality to each of the partitions.

- Operating System virtualization: - It deals with use of software or applications to allow hardware to run multiple instances of several systems simultaneously. These systems are isolated and independent of each other.

\subsection{Issues in Cloud Computing}

(i) Data Protection: - Data Security is one of major element that needs to be taken care of. Cloud vendors fear of losing confidential and identity of their consumers. In cloud model, service providers are responsible for maintaining data security and enterprises have to believe them.

(ii) Data Recovery and Availability: - All applications are designed by considering some laws or rules that are called as Service level agreements (SLA's). There are teams designed to support data availability at anytime. These teams perform following tasks:

- Data Replication

- $\quad$ System monitoring

- Maintenance

- Recovery from failure

(iii) Management Capabilities: - Although there are many multiple cloud providers, but management scale is not satisfactory. There is great need to improve on scalability and balancing features.

(iv) Regulatory and Compliance Standards: - In most of developing countries, cloud computing is still in infancy stage. There are various standards that are not known to people and their effect of using them.

\subsection{Challenges in Virtualization}

The top most issues that are governing every industry/ organizations are as follows:

- Virtualization backup and recovery: - Recovery from virtualized servers is little bit different from recovery from normal servers and databases. In virtualized servers, performing frequents backup may lead to loss of data from cloud. Although there are few backup vendors like Virtual Disk Development Kit (VDDK) for performing secure recovery but they are expensive and unavailable most of times.

- VM Sprawl: - Easy provision of virtual machines may lead to out of control of pop-up blockers on 
various cloud sites. The blockers will allow ads on sites simultaneously which leads to lack of management and handling of client requests.

- Virtual capacity planning: - To estimate future behaviors of resources, building budget and acquisition strategies, there are need to manage data efficiently by considering various factors like CPU memory usage, disk space and I/O functions.

- VM Stall: - Most companies are working on virtualized servers. Large number of virtual machines is increasing day by day due to virtualized deployment. VM Stall occurs due to lack of trust in administrators and performance management.

- Building a Private cloud: - Deploying services from virtual machines to private cloud is cumbersome task. It requires lot of infrastructure, geographical set-up and high investment. Also, there are no well defined strategies for building private cloud yet.

3. LAYOUT OF SYSTEMATIC MODEL FOR ACCESSING DATA SERVICES IN CLOUD BY USING MEMORY VIRTUALIZATION TECHNIQUE

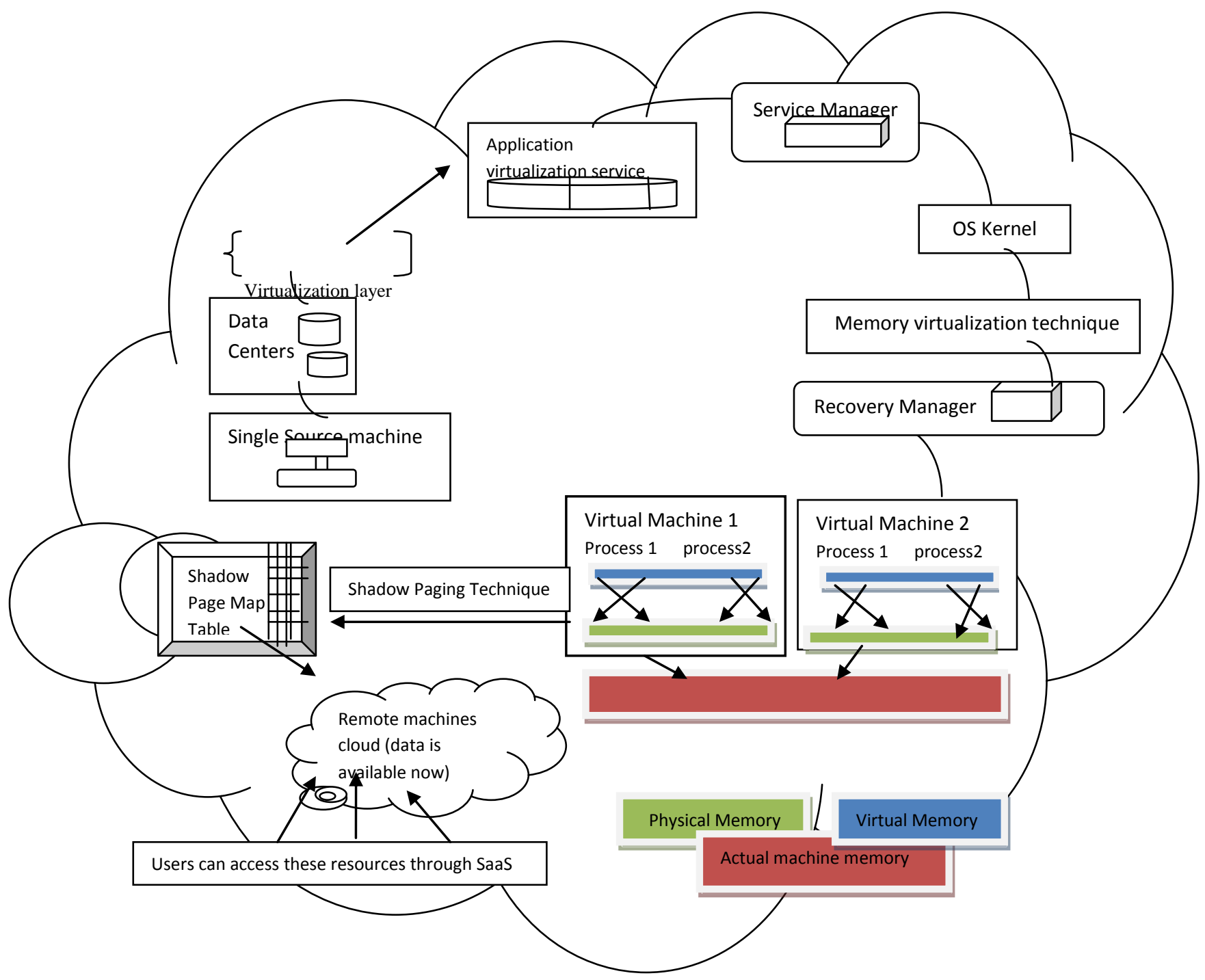

Fig 2: Systematic architecture employing Desktop-as-a-Service for accessing data from cloud using Memory Virtualization Technique 


\subsection{Features of proposed model}

(a) There is one single physical source machine that is connected to data center. The resources from these centers are allowed to enter virtualization cloud.

(b) Virtualization allows data to be transmitted to multiple virtual machines for performing fast access

of data. Servers connected to data center are allowed to enter virtualization environment.

(c) They are passed to application virtualizations service that follows Service Level Agreements (SLA's) factors like reliability, throughput, and durability etc thus reducing SLA violation

(d) Service Manager is used that monitors the flow of resources arriving through application service layer. It monitors client request.

(e) All these requests are sent to operating system for further processing and computation but the requests may or may not suit to hardware specifications.

(f) Here comes role of memory virtualization technique. It maps physical memory and virtual memory into actual machine memory.

(g) So, there is no matter of incompatibility between machine memory and virtual memory.

(h) After mapping of resources and instructions, it uses another technique called Shadow paging technique. With this technique, data is made available in remote cloud from which users can access data.

(i) Different user clients can now access these resources through SaaS.

\subsection{Evaluation of memory addresses using Shadow Paging Technique}

If in a system, fault occurs due to failure then there is provision of recovery manager in memory virtualization technique which applies various recovery techniques to have the accurate value in spite of failure in system.

Shadow Paging is one of recovery techniques that work implicitly according to memory status. After mapping of instructions from virtual to actual machine, it divides virtualized database into pages and its address is maintained in page map table. Whenever any update operation has to be performed, then the data will be copied to new location and its address will be maintained in SHADOW PAGE MAP TABLE.

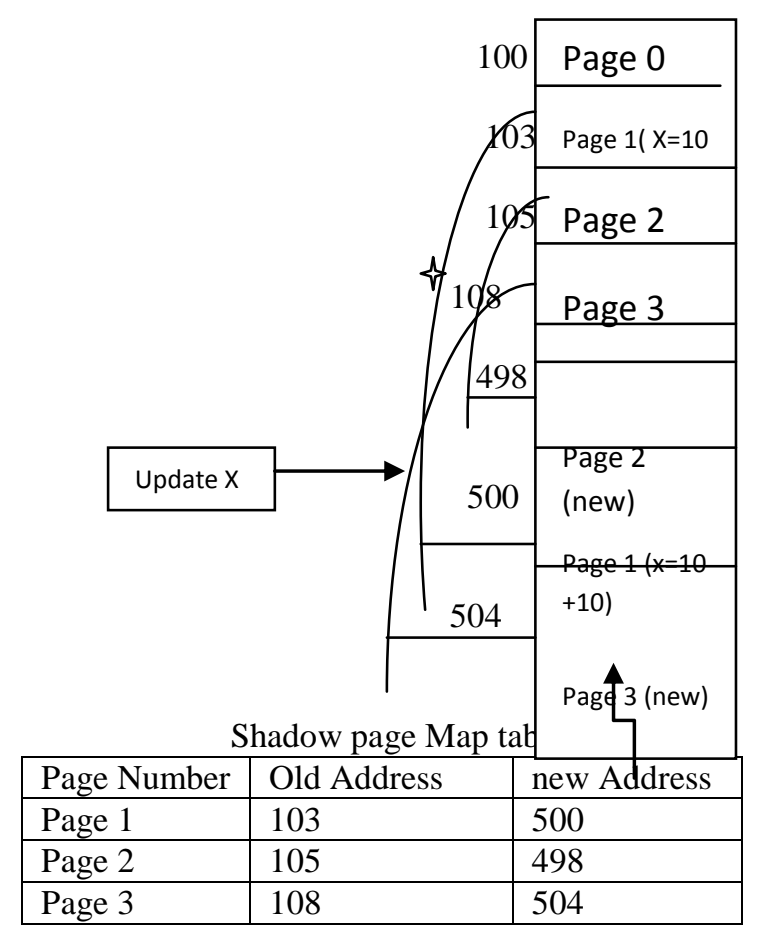

Figure 3: Shadow paging technique

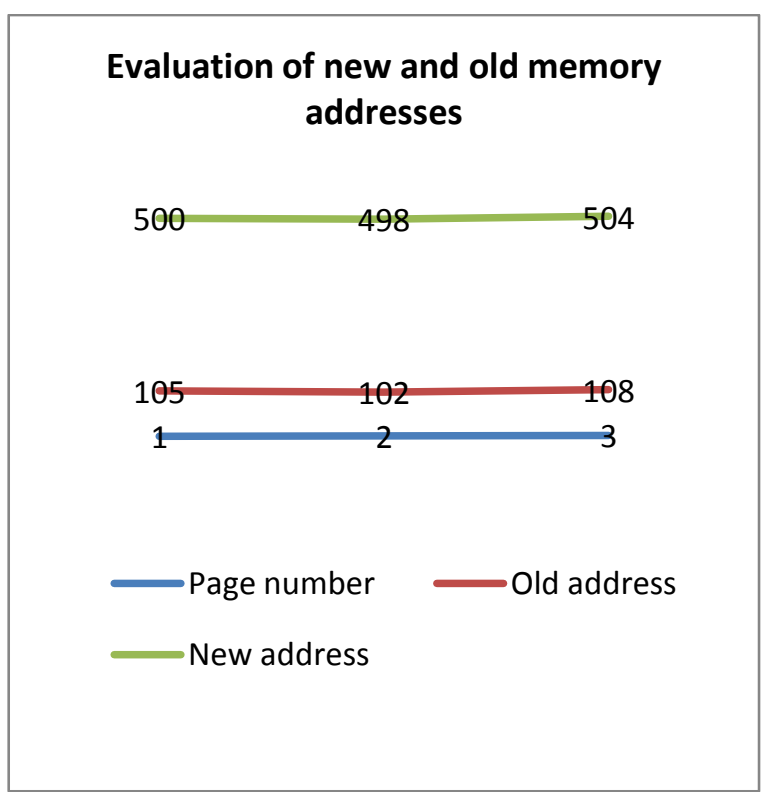

Figure 4: Analyzed results using shadow paging virtualization technique

\section{CONCLUSION}

Accessing data and resources from cloud is a task of difficulty and time consuming according to traditional technology methods and desktop-as-a-service. At that time, there were no wide areas of technology, lack of dynamic and distributed environment, less capability of data servers, less intensity of bandwidth index and many more bad factors. All these factors acted as obstructers in development of cloud environment.

The concept of virtualization has greatly changed the way of accessing data in cloud in spite of various factors. With the help of virtualization, it is possible to access data from cloud by using some of its virtualization techniques like para- 
virtualization, memory virtualization, hardware virtualization etc. The paper has shown model for accessing desktop-as-aservice using memory virtualization technique in cloud environment. The technique proves to have higher data efficiency with less percentage of violation of enterprises agreements.

The paper also shows recovery technique to save resources in case of failure in system. It is done by shadow paging technique. Overall memory virtualization technique is very useful to achieve efficient accessing of resources.

\section{REFERENCES}

[1]. Quiroz A, Kim H, Parashar M, Gnanasambandam N, Sharma N, 2009, "Towards workload provisioning for enterprise grids and clouds", IEEE/ACM international conference on grid computing. pp 50-57.

[2]. Soramichi Akiyama, Takahiro Hirofuchi, Ryousei Takano, Shinichi Honiden, 2012, "MiyakoDori: A Memory Reusing Mechanism for Dynamic VM Consolidation", Fifth International Conference on Cloud Computing, IEEE 2012.

[3]. Abirami S.P., Shalini Ramanathan, 2012 "Linear Scheduling Strategy for Resource allocation in CloudEnvironment",International Journal on Cloud Computing and Architecture vol.2, No.1, February.

[4]. Christopher Clark, Keir Fraser, Steven Hand, Jacob Gorm Hanseny, Eric July, Christian Limpach, Ian Pratt, Andrew Warfield, 2005, "Live Migration of Virtual Machines", 2nd Symposium on Networked Systems Design and Implementation (NSDI), May
[5]. Lien Deboosere, Bert Vankeirsbilck ,Pieter Simoens, Filip DeTurck , Bart Dhoedt and Piet Demeester, "Efficient resource management for virtual desktop cloud computing", Springer2012

[6]. Gartner's 2008 Data Center Conference Instant Polling Results:Virtualization Summary - March 2, 2009

[7]. L. Wu, S. K. Garg, and R. Buyya. SLA-based resource allocation for software as a service provider (SaaS) in cloud computing environments. In C Grid 2011, 11th IEEE/ACM International Symposium on Cluster, Cloud and Grid Computing, Newport Beach, CA, USA, May 2011

[8]. Rao Mikkilineni, Vijay Sarathy "Cloud Computing and Lessons from the Past", Proceedings of IEEE WETICE 2009, First International Workshop on Collaboration \& Cloud Computing, June 2009

[9]. V. Krishna Reddy, B. Thirumal Rao, Dr. L.S.S. Reddy, P.Sai Kiran "Research Issues in Cloud Computing " Global Journal of Computer Science and Technology, Volume 11,Issue 11, July 2011

[10]. Dan Sullivan, "The Definitive Guide to Cloud Computing", Realtime Publishers

[11]. Gagandeep Singh Narula, "Improving Statistical Multimedia Information Retrieval (MIR) Model by using Ontology" 'International Journal of Computer Applications (IJCA)' USA, Vol. 94 No 2. May 2014 page no. 35-45 ISSN No. (Online) 0975-8887 